\section{Reduced intensity transplantation for primary immunodeficiency disorders}

\author{
Paul Veys \\ Department of BMT, Great Ormond \\ Street Hospital for Children NHS Trust, \\ London, UK
}

\section{Abstract}

Studies so far indicate that reduced intensity transplantation (RIT) may have an important role in treating patients with primary immunodeficiency disease (PID). Unlike more standard approaches, such regimens can be used without severe toxicity in patients with severe pulmonary or hepatic disease. RIT also offers the advantage that long-term sequelae such as infertility or growth retardation may be avoided or reduced. RIT appears to be most appropriate for those patients with significant co-morbidities (eg T cell deficiencies) and those undergoing unrelated donor haematopoietic cell transplantation. More studies are required using pharmacokinetic monitoring (eg busulphan, treosulfan and alemtuzumab) and varying stem cell sources to optimise graft vs marrow reactions and minimise graft vs host disease. In certain PID patients RIT will be the "first step" towards establishing donor cell engraftment; second infusions of donor stem cells, donor lymphocyte infusions, or a second myeloablative HCT, which appears to be well tolerated, may be required in some patients with low level donor chimerism or graft rejection.

\section{Introduction}

Many children with primary immunodeficiency disease (PID) have significant co-morbidities at the time of haematopoietic cell transplantation (HCT) and conventional myeloablative preparation may be associated with significant treatment-related toxicity as well as longterm sequelae. Over the past decade reduced intensity transplantation (RIT) has become a well established approach in adult patients with malignant disease, extending curative HCT to older individuals and patients with co-morbidities otherwise ineligible for myeloablative procedures.1,2,3 RIT is therefore an attractive option for children with PID, and particularly as there is no requirement for high dose chemotherapy to eradicate malignancy; graft failure, however, may be a concern in certain groups as many patients with non-malignant disorders will not have received prior chemotherapy.

\section{Mechanism of RIT}

Conventional HCT prevents rejection by the use of supralethal chemotherapy to remove host-versus-graft $(\mathrm{HvG})$ reactions, and create marrow space, often achieving full donor chimerism in the early months post HCT. RIT prevents rejection by the use of pre +/- post HCT immunosuppression to achieve tolerance and a graft-versus-marrow (GvM) reaction to create space. In this setting a stable mixed chimerism is often achieved which may be converted to full donor chimerism, if required, by tailing immunosuppression or donor lymphocyte infusions (DLI). Unlike the situation in malignant disease, stable mixed chimerism in the affected cell lineage is usually sufficient to cure genetic diseases.

Two general approaches have been used to develop RIT regimens. 4,5

So-called reduced intensity conditioning (RIC) protocols (Figure 1) have been developed by replacing myeloablative agents with more immunosuppressive and less myelosuppressive properties. ${ }^{6,7}$ Such protocols, nevertheless, still contain agents capable of ablating stem cells eg busulphan or melphalan, but at a reduced dose compared to conventional HCT. In contrast, regimens with minimal toxicity or minimal intensity conditioning (MIC) (Figure 1) are truly non-myeloablative and contain only immunosuppressive agents. These latter regimens, developed in animal models, initially used irradiation to induce a degree of immunosuppression pre-transplant, followed by post-transplant immunosuppression given to control residual host as well as newly infused donor, alloreactive T cells. ${ }^{8}$ By definition MIC procedures have been associated with less toxicity than RIC HCT, however, as MIC relies solely on a GvM reaction to make marrow space, there is a suggestion that MIC HCT may be associated with an increased incidence of graft-versus-hostdisease (GvHD), particularly chronic (c)GvHD, and especially in the unrelated donor setting.

\section{RIT protocols for PID}

Most reduced and minimal intensity protocols are based around the purine analogue fludarabine which has profound immunosuppressive properties (Figure 1). RIC protocols combine fludarabine with a marrow ablative agent either melphalan, busulphan or treosulphan, and MIC protocols combine fludarabine with non-marrow ablative low dose radiation or cyclophosphamide.

\section{Reduced intensity conditioning protocols for PID}

\section{Fludarabine/melphalan}

Our group recently updated their series, reporting 113 patients with PID who had undergone RIT between 1998 and 2006..$^{9}$ The majority of patients (93/113) received a RIC reg-
Correspondence: Paul Veys, Department of BMT, Great Ormond Street Hospital for Children NHS Trust, Great Ormond Street, London WC1N 3JH, UK.

E-mail: veysp@gosh.nhs.uk

Key words: immunodeficiency disorders, reduced intensity transplantation.

Received for publication: 4 May 2011.

Accepted for publication: 4 June 2011.

This work is licensed under a Creative Commons Attribution NonCommercial 3.0 License (CC BYNC 3.0).

(C) Copyright P. Veys, 2011

Licensee PAGEPress, Italy

Pediatric Reports 2011; 3(s2):e11

doi:10.4081/pr.2011.s2.e11

imen consisting of Campath $1 \mathrm{H} 1 \mathrm{mg} / \mathrm{kg}$ (Alemtuzumab), fludarabine $150 \mathrm{mg} / \mathrm{m}^{2}$, melphalan $140 \mathrm{mg} / \mathrm{m}^{2}$ (FMC-RIC). At a median follow-up of 2.9 years (range 2 months to 8 years) the overall survival (OS) for these patients was $82 \%(93 / 113)$ and $91 / 133$ (81\%) had stable donor engraftment. The survival curve following RIC HCT for the major PID diseases compared to that following MAC HCT in other European centers is shown in Figure 2.

Long-term chimerism (median follow-up 4.6 years, range 6 months to 10.6 years) has also been examined in 118 children with PID receiving FMC-RIC HCT in London [Rao et al. 2010 manuscript in preparation]. After prolonged follow-up donor chimerism was low $(<50 \%)$ in $24 / 118(20 \%)$ patients, 5 patients have required a second myeloablative (MAC) HCT, one required a CD34+ cell top-up, two patients were given DLI, one patient with WAS underwent a splenectomy. Twenty one of these 24 patients are currently alive and well with stable engraftment. Two patients have died, one following $2^{\text {nd }}$ HCT and one from progressive disease, and one patient has continuing poor immune reconstitution. Almost all patients developing low level donor chimerism received BM rather than PBPCs as stem cell source and MSD and MFD had more low MC than MUDs and mMUDs (30\% and $28 \%$ vs $18 \%$ and $11 \%)$. Very low (<10\%) donor chimerism was almost entirely limited to the myeloid series. Cyclosporin withdrawal appeared to have a positive effect on lymphoid chimerism but not on myeloid engraftment. Lymphoid chimerism changed little after the first year but myeloid chimerism did decrease further after one year in a few patients. Consequently 5 years following RIC HCT for PID just under $10 \%$ of patients have required a second procedure.

Shenoy and colleagues ${ }^{10}$ used FMC-RIC HCT in 16 patients with non-malignant disorders 


\section{A hierarchy of conditioning intensity}

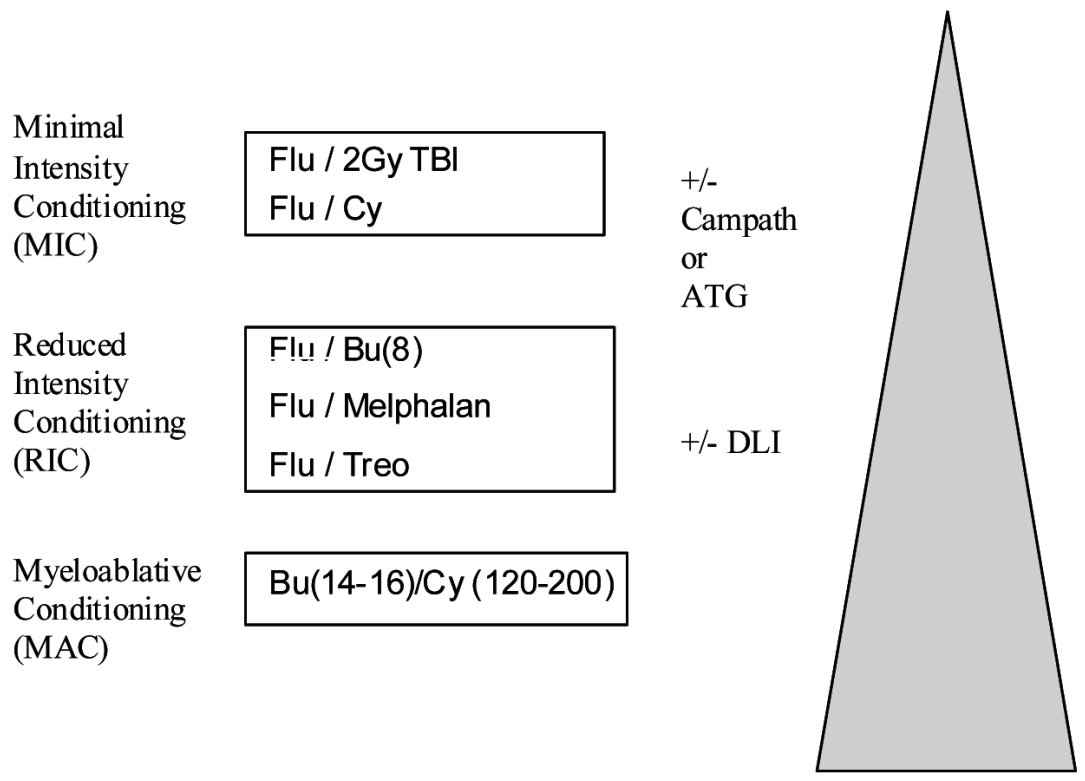

Figure 1. A hierarchy of commonly used minimal intensity (MIC), reduced intensity (RIC) and myeloablative conditioning (MAC) regimens in PID patients; Gy, gray; Flu, fludarabine; cyclo, cyclophosphamide; BU8, busulfan $8 \mathrm{mg} / \mathrm{kg}$; BU14-16, busulfan 14-16 mg/kg; CY120-200, cyclophosphamide 120-200 mg/kg; DLI, donor lymphocyte infusion; ATG, antithymocyte globulin. Bone Marrow Transplant 2008;41(2):174, with permission.
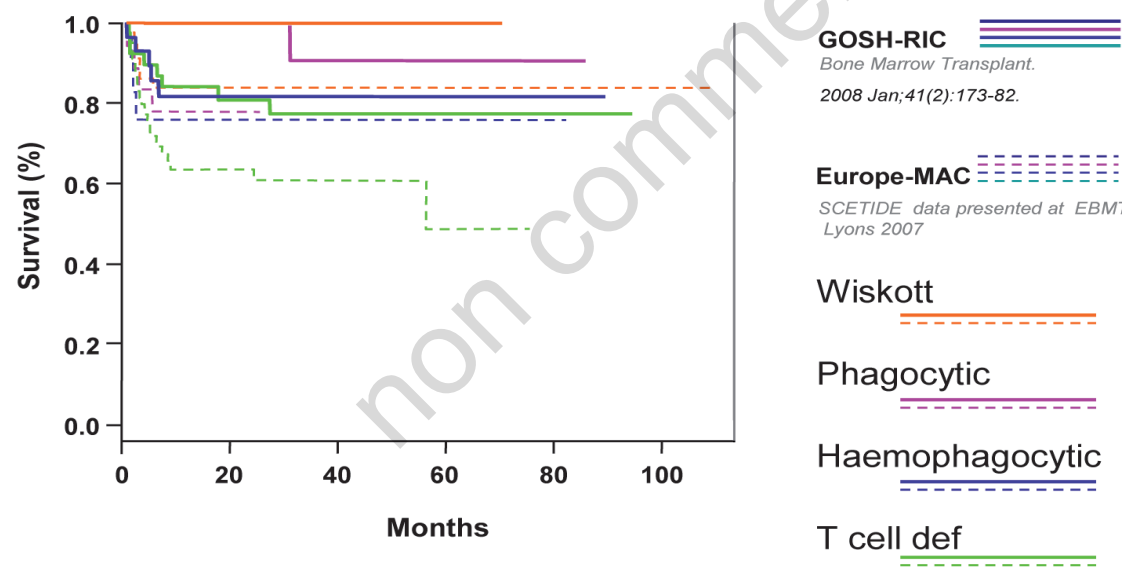

GOSH-RIC, Great Ormond Street Hospital reduced intensity conditioning HCT.

Europe MAC, myeloablative HCT performed in European centres. Def, deficiency.

Figure 2. Improvement in outcome of stem cell transplantation for T-cell immune deficiency.

including 2 PID patients, but in their series administered Campath 1H 33 or $48 \mathrm{mg}$ total dose early pre HCT from day -21 to day -19 . All fourteen evaluable patients had complete or high level (> 50\%) donor chimerism in all lineages, suggesting that lower doses or administration of Campath 1H away from the graft may increase donor chimerism in the HLA-matched setting. Further studies are underway to examine whether Campath levels taken on or around day zero may predict graft outcome in these patients: ie high levels predicting for slow immune reconstitution and viral infections and low levels for GVHD and complete donor chimerism. These results may better help to define the optimal method of delivering Campath in the RIT setting.

The benefit from FMC-RIC HCT was most evident in children over 1 year of age. For SCID patients under 1 year, TRM remained high even with RIC HCT (24\% in the London series) with $17 / 30$ patients (57\%) needing intensive care management post HCT; this compares to 26/118 (22\%) in the group $>1 \mathrm{yr}$ of age $(\mathrm{P}<0.0001)$.

\section{Fludarabine/busulphan}

The most notable study exploring RIC HCT in PID using fludarabine and low dose busulfan has been reported in chronic granulomatous disease (CGD) patients. Intravenous busulfan 8-10 mg/kg (adjusted with busulphan kinetics in pediatric patients), fludarabine 180 $\mathrm{mg} / \mathrm{m}^{2}$ and ATG $40 \mathrm{mg} / \mathrm{kg}$ was used with matched donors $(\mathrm{MSD}=5, \mathrm{MUD}=3)$ in 8 highrisk CGD patients and led to $90-100 \%$ donor chimerism in all cases at a median follow-up of 26 months. ${ }^{11,12}$ This is despite the use of BM in $7 / 8$ cases. Seven patients were alive and well and all active inflammatory and infectious foci were cured. One adult patient who had received PBSC from a CMV negative MUD died on day +150 of CMV pneumonitis. This cohort has recently been updated and 27/28 CGD patients survive following RIT HCT with MSD(14) and MUD/mMUD(14) donors [Gungor 2010 personal communication ].

\section{Fludarabine/treosulphan}

Treosulfan (L-treitol-1,4-bis-methanesulfonate) is the pro-drug of L-epoxybutane, a water soluble bifunctional alkylating agent with myeloablative and immunosuppressive properties and has been shown to provide effective HCT conditioning with reduced risk of toxicities, particularly veno-occlusive disease (VOD), when compared to busulfan.13 Two centers in the UK have recently examined the outcome in 70 children with PID undergoing HCT with treosulfan based regimens. ${ }^{14}$ Children received $42 \mathrm{~g} / \mathrm{m}^{2}$ or $36 \mathrm{~g} / \mathrm{m}^{2}$ of treosulfan with cyclophosphamide $200 \mathrm{mg} / \mathrm{kg}$ (30) or fludarabine $150 \mathrm{mg} / \mathrm{m}^{2}$ (40), with alemtuzumab in most. Median age at transplant was 8.5 months (1.2 to 175$)$; forty-six (66\%) patients were 12 months or younger. Overall survival was $81 \%$, and equivalent in those aged less or greater than one year. The combination of treosulfan with fludarabine was associated with lower toxicity and improved $\mathrm{T}$ cell chimerism. There were significantly fewer under 1 year olds admitted to intensive care following HCT in this study (12/44) compared to those who received the combination of fludarabine and melphalan (17/30 $\mathrm{P}=0.0155)$.

Long-term follow up is required to determine gonadotoxic effects, but the combination 
of fludarabine with treosulfan is a good choice of conditioning for HCT in PID of any age.

\section{Minimal intensity conditioning}

\section{Fludarabine/low dose TBI}

The Seattle group investigated a MIC regimen in 14 patients (12 children and 2 adults) with PID and coexisting infections, organ toxicity or other factors precluding conventional HCT. 15 The majority of patients received 200 cGy TBI plus Flu $\left(30 \mathrm{mg} / \mathrm{m}^{2}\right.$ per day; $\mathrm{x} 3$ days -4 to -2 ) as conditioning and all patients received HLA-matched grafts with intensive post-graft immunosuppression with CsA/MMF. No serotherapy was given. Thirteen patients established mixed $(n=5)$ or full $(\mathrm{n}=8)$ donor chimerism and one rejected the graft. OS at 3 years was $62 \%$ with a TRM of 23\%. Eight of ten evaluable patients had correction of immune deficiency with stable donor engraftment. However, there was a high rate of GVHD with 11/14 developing significant aGVHD (mostly grade II) and extensive cGVHD in eight patients, reflecting both the use of peripheral blood as the stem cell source and the absence of serotherapy. This approach was associated with a lower incidence of viral infections/reactivations, notably EBV, than RIC regimens utilizing serotherapy, however, the high incidence of cGVHD would be a significant obstacle to broader use of this regimen in children with non-malignant disorders.

Fludarabine/cyclophosphamide/mabs

Our group has explored a MIC protocol combining fludarabine $\left(30 \mathrm{mg} / \mathrm{m}^{2} \times 5\right.$ day -8 to -4$)$ and low dose cyclophosphamide $\left(300 \mathrm{mg} / \mathrm{m}^{2} \mathrm{x}\right.$ 4 on day -7 to -4 ) with two rat anti-C45 monoclonal antibodies YTH 24.5/YTH 54.12 for additional myelosuppression, and serotherapy with Campath $1 \mathrm{H}$ either $0.6 \mathrm{mg} / \mathrm{kg}$ or $0.3 \mathrm{mg} / \mathrm{kg}$ with UD or MSD respectively.16 Patients were at particularly high risk from HCT related toxicity even with RIC protocols due to severe pre-existing organ toxicity, age < 1 year, or the presence of DNA/telomere repair disorders. In total 16 patients underwent MIC HCT from MSD (5), MUD (9) and mMUD (2). Conditioning was well tolerated with only 2 cases of grade 3 and no grade 4 toxicity. Six out of 16 patients (38\%) developed significant aGVHD (3 grade II skin and 3 grade III skin/gut). Five of 16 patients (31\%) developed cGVHD (limited in 3 and extensive in 2) which has resolved in all cases. Of note, the incidence of GVHD was reduced when BM was used as stem cell source (2/10 BM recipients compared with 4/4 evaluable PBSC recipients developed aGVHD > grade II). Similarly the incidence of cGVHD was lower in recipients of BM (2/10) as compared to PBSC (3/4). At a median of 9.5 days (range 1-15), 16/16 patients had a neutrophil count $>0.5 \times 10^{9} / \mathrm{L}$. One patient failed to engraft and had autologous recovery and one patient who received a mismatched CB engrafted with stable mixed chimerism after a very extended period. Donor chimerism was $100 \%$ in $3 / 4$ PBSC recipients with one $\mathrm{PBSC}$ recipient rejecting the graft. Three out of $10 \mathrm{BM}$ recipients achieved $100 \%$ donor chimerism, 3 achieved stable high level MC in both mononuclear and granulocyte lineages and 3 achieved donor T cell chimerism without susutained myeloid chimerism. One achieved very low level donor chimerism and required a second SCT. At a median of 37 months post HCT 13/16 patients in this high risk cohort were alive and cured from their underlying disease. In terms of OS, SCID patients $<1$ year of age appeared to gain particular benefit from this MIC HCT protocol (Figure 3).

\section{Stem cell source}

The balance of HvG and $\mathrm{GvH} / \mathrm{GvM}$ reflects the complex interactions of stem cell source with disease type, conditioning regimen, serotherapy, graft content (CD34+, CD3+, NKKIR alloreactivity) and GvHD prophylaxis, and is more finely balanced in RIT than MAC HCT. Early warning of impending graft rejection may be gained from recipient chimerism status in NK-cells on day +2817 or early in- creasing MC > 30\% host cells, ${ }^{18}$ and might prompt timely intervention by withdrawl of immune suppression or DLI.

In contrast to reports in adults, treatmentrelated mortality, treatment failure, and mortality were higher after MAC transplantation in children using PBPCs as opposed to BM. ${ }^{19}$ Following RIT, the use of PBSCs appears to be associated with improved donor chimerism in recipents with PID, ${ }^{14,15}$ but at the cost of increased rates of GvHD, particularly in mMUD; in London PBPCs are advocated for 10/10 HLA matched related and unrelated donors following RIT HCT and new approaches are being pursued for mMUDs (Rao et al., 2010 manuscript in preparation). In the UK, the use of GCSF is not advocated in sibling donors $<16$ yrs of age, and as the combination of RIT and MSD BM often leads to low level donor chimerism in PID patients, MAC is generally preferred for this group.

There has been increasing interest in the use of UCB in PID but using largely myeloablative preparations. ${ }^{20} \mathrm{UCB}$ has the advantage of immediate access and a lower rate of GvHD making it particularly attractive stem cell source for children with PID. There is limited experience with the use of RIT and UCB in children with PID. Fifteen of 17 patients who

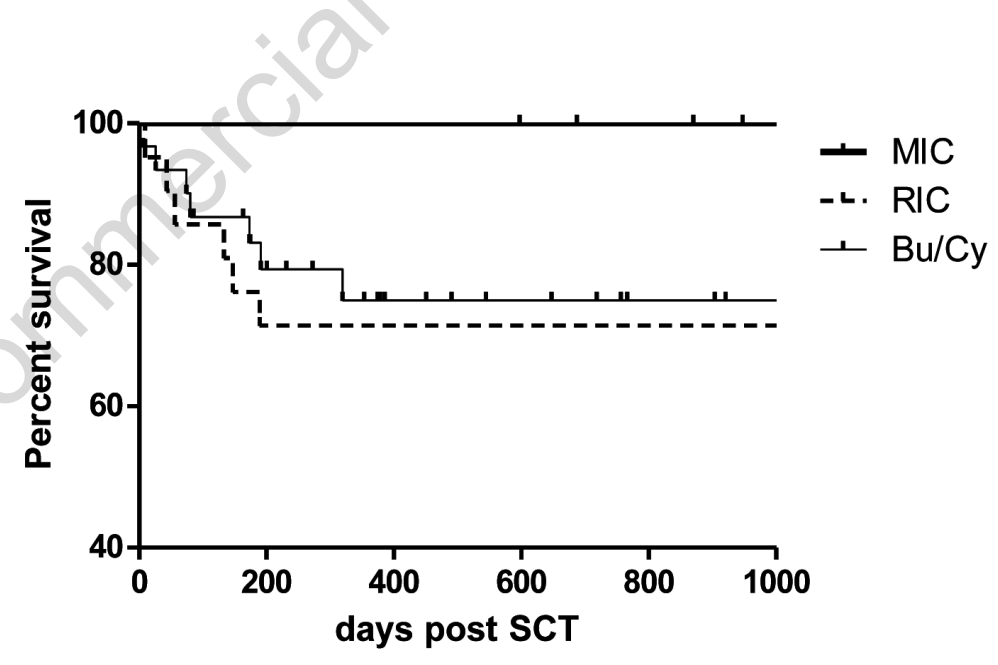

$\begin{array}{lrrrrrr}\text { Number at risk } & & & & & & \\ \text { MIC } & 8 & 8 & 8 & 8 & 8 & 8 \\ \text { RIC' } & 21 & 15 & 15 & 15 & 15 & 15 \\ \text { Bu/Cy } & 31 & 25 & 24 & 24 & 24 & 24\end{array}$

Figure 3. Comparison of disease-free survival of SCID patients $<1$ year of age transplanted using anti-CD45 MAb-based MIC, fludarabine/melphalan based RIC and busulphan/cyclophosphamide conditioning. Reproduced with permission. Kaplan-Meier curves showing disease-free survival (days) of SCID patients age $<1$ year conditioned with (1) CD45 MAb-based MIC regimen (n= 8, DFS $100 \%)$ (2) fludarabine/melphalan-based RIC regimen $(\mathrm{n}=21$, DFS $71.4 \%)$ and (3) busulphan/cyclophosphamide-based conditioning $(n=31$, DFS $77.4 \%)$. The cohort conditioned with CD45-based MIC was transplanted between 2003-2007 (donor source 63\% MUD, 25\% MMUD, 13\% MSD, 37\% B $^{\text {neg }}$ phenotype), the cohort conditioned with fludarabine/melphalan was transplanted between 1999-2003 (donor source 81\% MUD, 19\% MMUD, 57\% $\mathrm{B}^{\text {neg }}$ phenotype) and the cohort transplanted with busulphan/cyclophosphamide was transplanted between 2003-2005 (donor source 57\% MUD, 30\% MSD, 13\% MFD, 46\% Bneg phenotype). ${ }^{16}$ 
had treosulfan based RIC HCT in UK survived; 14 the omission of serotherapy in this group led to high levels of donor chimerism and rapid reconstitution of CD4+ lymphocytes and anti-viral immunity within 1-2 months of HCT, making this combination particularly suitable for PID.

\section{Conclusions}

RIT has an important role in treating patients with PID, and appears to be most appropriate for those patients with significant comorbidities (eg T cell deficiencies) and those undergoing UD HCT; the optimal choice of protocol and stem cell source remains to be elucidated. RIT also offers the advantage that longterm sequelae such as infertility or growth retardation may be avoided or reduced. In certain PID patients RIT will be the "first step" towards establishing donor cell engraftment, second infusions of donor stem cells, donor lymphocyte infusions, or a second myeloablative HCT may be required.

\section{References}

1. Alousi A, de Lima M. Reduced-intensity conditioning allogeneic hematopoietic stem cell transplantation. Clin Adv Hematol Oncol 2007;5:560-70.

2. Barrett AJ, Savani BN. Stem cell transplantation with reduced-intensity conditioning regimens: a review of ten years experience with new transplant concepts and new therapeutic agents. Leukemia 2006;20:1661-72.

3. Rezvani AR, Storb R. Using allogeneic stem cell/T-cell grafts to cure hematologic malignancies. Expert Opin Biol Ther 2008;8:161-79.

4. Woolfrey A, Pulsipher MA, Storb R. Nonmyeloablative hematopoietic cell transplant for treatment of immune deficiency.
Curr Opin Pediatr 2001;13:539-45.

5. Satwani P, Cooper N, Rao K, et al. Reduced intensity conditioning and allogeneic stem cell transplantation in childhood malignant and nonmalignant diseases. Bone Marrow Transplant 2008;41:173-82.

6. Slavin S, Nagler A, Naparstek E, et al. Nonmyeloablative stem cell transplantation and cell therapy as an alternative to conventional bone marrow transplantation with lethal cytoreduction for the treatment of malignant and nonmalignant hematologic diseases. Blood 1998;91:756-63.

7. Champlin R, Khouri I, Shimoni A, et al. Harnessing graft-versus-malignancy: non-myeloablative preparative regimens for allogeneic haematopoietic transplantation, an evolving strategy for adoptive immunotherapy. Br J Haematol 2000;111:18-29.

8. Storb R, Yu C, Wagner JL, et al. Stable mixed hematopoietic chimerism in DLAidentical littermate dogs given sublethal total body irradiation before and pharmacological immunosuppression after marrow transplantation. Blood 1997;89:3048-54.

9. Satwani P, Cooper N, Rao K, et al. Reduced intensity conditioning and allogeneic stem cell transplantation in childhood malignant and nonmalignant diseases. Bone Marrow Transplant 2008;41:173-82.

10. Shenoy S, Grossman WJ, DiPersio J, et al. A novel reduced-intensity stem cell transplant regimen for nonmalignant disorders. Bone Marrow Transplant 2005;35:345-52.

11. Güngör T, Halter J, Klink A, et al. Successful low toxicity hematopoietic stem cell transplantation for high-risk adult chronic granulomatous disease patients. Transplantation 2005;79:1596-606.

12. Gungor T, Halter J, Stussi G, et al. Successful busulphan-based reduced intensity conditioning in high-risk paediatric and adult chronic granulomatous disease - The Swiss experience. Bone Marrow Transplant 2009;43:S75.

13. Greystoke B, Bonanomi S, Carr TF, et al. Treosulfan-containing regimens achieve high rates of engraftment associated with low transplant morbidity and mortality in children with non-malignant disease and significant co-morbidities. Br J Haematol 2008;142:257-62.

14. Slatter M, Rao K, Amrolia P et al. Treosulfan-based conditioning regimens for haematopoietic stem cell transplantation in children with Primary Immunodeficiency (PID): UK experience. Blood 2011; 117:4367-75.

15. Burroughs LM, Storb R, Leisenring WM, et al. Intensive postgrafting immune suppression combined with nonmyeloablative conditioning for transplantation of HLAidentical hematopoietic cell grafts: results of a pilot study for treatment of primary immunodeficiency disorders. Bone Marrow Transplant 2007;40:633-42.

16. Straathof KC, Rao K, Eyrich M, et al. Haemopoietic stem cell transplantation with antibody-based minimal intensity conditioning regimen for children with severe organ toxicity or DNA repair disorders. Lancet 2009;374:912-20.

17. Matthes-Martin S, Lion T, Haas OA, et al. Lineage-specific chimaerism after stem cell transplantation in children following reduced intensity conditioning: potential predictive value of NK cell chimaerism for late graft rejection. Leukemia 2003;17:1934-42.

18. Ozyurek E, Cowan MJ, Koerper MA, et al. Increasing mixed chimerism and the risk of graft loss in children undergoing allogeneic hematopoietic stem cell transplantation for non-malignant disorders. Bone Marrow Transplant 2008;42:83-91.

19. Eapen M, Horowitz MM, Klein JP, et al. Higher mortality after allogeneic peripheral-blood transplantation compared with bone marrow in children and adolescents: the Histocompatibility and Alternate Stem Cell Source Working Committee of the International Bone Marrow Transplant Registry. J Clin Oncol 2004;22:4872-80.

20. Gennery AR, Cant AJ. Cord blood stem cell transplantation in primary immune deficiencies. Curr Opin Allergy Clin Immunol 2007;7:528-34. 